\title{
Participação da Família no Processo Decisório de Limitação de Suporte de Vida: Paternalismo, Beneficência e Omissão*
}

\author{
Family Participation in the Decision Making Process for Life Support \\ Limitation: Paternalism, Beneficence and Omission
}

Patrícia Miranda Lago', Daniel Garros², Jefferson P. Piva ${ }^{3}$

\section{RESUMO}

JUSTIFICATIVA E OBJETIVOS: Analisar e discutir os aspectos médicos relacionados a participação da família nos cuidados de final de vida oferecidos a crianças internadas em unidades de tratamento intensivo pediátrico (UTIP).

CONTEÚDO: Foram selecionados artigos sobre cuidados de final de vida publicados nos últimos 20 anos na PubMed, MedLine e LILACS com ênfase nos estudos sobre morte em UTIP no Brasil, América Latina, Europa e Norte América, usando as palavras-chave: morte, bioética, tratamento intensivo pediátrico, pacientes terminais, limitação de suporte de vida, entrevista com familiares.

CONCLUSÕES Vários estudos têm demonstrado a importância da participação da família no processo decisório de imitação de suporte de vida. Em nosso meio a participação da família neste processo ainda é pouco

1. Pediatra Intensivista do Hospital de Clinicas de Porto Alegre. Doutora em Pediatria pela PUCRS

2. Professor Adjunto de Pediatria, Stollery Children's Hospital, Dept Critical Care, University of Alberta, Edmonton, AB, Canadá

3. Professor Adjunto dos Departamentos de Pediatria da PUCRS e da UFRGS. Chefe Associado da Unidade de Terapia Intensiva Pediátrica do Hospital São Lucas-PUCRS. Secretário da World Federation of Pediatric Intensive and Critical Care Societies (WFPICCS)

*Recebido do Departamento de Pós-Graduação em Pediatria da Pontifícia Universidade Católica do Rio Grande do Sul (PUCRS), Porto Alegre, RS

Apresentado em 31 de agosto de 2007

Aceito para publicação em 18 de setembro de 2007

Endereço para correspondência:

Dra. Patrícia Miranda Lago

Rua Furriel Luis Vargas 238/201

90470-130 Porto Alegre, RS

Fone/Fax: (51) 3333-4462 - Cel. 9678-0431

E-mail: lagopatricia@terra.com.br

(C)Associação de Medicina Intensiva Brasileira, 2007 estimulado e valorizado, oscilando entre $20 \%$ e $55 \%$. Os autores apresentam uma seqüência de abordagem na definição de LSV com a família. Apesar da sua participação no processo de final de vida de pacientes terminais ter amparos ético, moral e legal e ser praticada em paises desenvolvidos, esta conduta é pouco adotada. Estudos devem realizados na tentativa de explicar esta dificuldade encontrada pelos intensivistas pediátricos brasileiros.

Unitermos: bioética, entrevista com familiares, limitação de suporte vital, morte, unidade de terapia intensiva pediátrica

\section{SUMMARY}

BACKGROUND AND OBJECTIVES: To analyze and discuss the medical aspects related to the family involvement in the decision making process regarding end of life care to children admitted to the pediatric intensive care unit (PICU).

CONTENTS: The authors selected articles on end-oflife care published during the last years searching the PubMed, MedLine and LILACS database, with special interest on studies of death conducted in pediatric intensive care units in Brazil, Latin America, Europe and North America, using the following keywords: death, bioethics, PICU, decision-making, terminal care, parents interview and life support limitation (LSL).

CONCLUSIONS: Several studies have demonstrated the relevance of the family participation in the decision making process regarding LSL. In our region the family participation in this process is not stimulated and valued, ranging from $20 \%-55 \%$. The authors present a practical sequence for discussing and defining LSL with the families. Despite of the family participation in the decision making process for LSL be legally, morally and ethically accepted in developed countries, this approach is adopted in a very few cases in our region. To explain this difficulty observed among the Brazilian pe- 
diatric intensivist, some studies should be conducted in our region.

Key Words: bioethics, death, decision-making, parents interview and life support limitation, pediatric intensive care unit.

\section{INTRODUÇÃO}

A morte em unidade de terapia intensiva pediátrica (UTIP) é menos freqüente que em UTI de adultos e a maioria das crianças que falecem nestas unidades é portadora de doença crônica ${ }^{1,2}$. Estima-se que mais de $50 \%$ das crianças com doenças terminais morrem dentro de um hospital e mais de $90 \%$ destas, após uma semana de hospitalização em UTIP ${ }^{3,4}$. No mundo ocidental, existe o consenso de que os cuidados de final de vida e a qualidade da morte são fundamentais no atendimento de crianças criticamente enfermas. Por estes motivos, o intensivista pediatra deve estar preparado para atender as necessidades destas crianças com doenças irreversíveis, oferecendo a melhor opção terapêutica que possa garantir uma morte sem sofrimento ${ }^{5-8}$.

O paciente pediátrico difere do adulto por não ter capacidade de decidir sobre as condutas que devem ser tomadas no seu final de vida. Sendo assim a família possui papel fundamental, já que defende o melhor interesse da criança. Após o consenso médico sobre a irreversibilidade, a família deve ser comunicada e convidada a participar de todos os passos que se seguem na definição dos planos de final de vida. Porém, esta conduta não é seguida em todos os paises ${ }^{9,10}$.

Os cuidados de final de vida de pacientes terminais passam por quatro etapas que devem ser seguidas em ordem. Na primeira etapa, se busca o consenso entre a equipe assistencial sobre a irreversibilidade da situação, só após a decisão consensual que se passa para a segunda fase, que é a comunicação para a família. Esta conversa deve ser feita em lugar tranqüilo, garantindo a privacidade do encontro. Geralmente, em UTIP, a família escolhe o profissional que tem maior proximidade com o paciente. A morte de uma criança desencadeia um luto profundo, por isto, dificilmente a família aceita no primeiro encontro o diagnóstico e prognóstico de doença irreversível. Na terceira etapa traçam-se junto com a família os planos de final de vida. Nesta fase, definem-se limitações de suporte de vida (LSV) e condutas que visem o conforto do paciente. Na quarta etapa, as metas escolhidas pela família junto com a equipe médica são aplicadas ${ }^{9}$.
Uma freqüente fonte de frustração das famílias que perderam um ente querido em UTI é de que "ninguém sentou e discutiu o fato de que o paciente estava na realidade morrendo". Palavras vagas como "pode ser que ele não vá melhorar" levam à confusão e a falsas esperanças. Se a família capta claramente a mensagem de que sua criança está morrendo, ela terá maior tempo para dedicar às despedidas, para contatar parentes distantes, perguntar coisas mais apropriadas aos cuidados necessários nessa fase, enfim, prepararse para a morte ${ }^{11}$.

\section{PARTICIPAÇÃO DA FAMÍLIA NO PROCESSO DE FI- NAL DE VIDA}

A participação da família na discussão e definição de metas de final de vida é reconhecida como de extrema importância por todos que trabalham em UTIP, no entanto, nem sempre os familiares são solicitados a participar ativamente no processo decisório ${ }^{12}$.

$\mathrm{Na}$ América Latina, nas poucas publicações disponíveis, observa-se que o envolvimento da família no processo decisório de LSV de suas crianças ainda é discreto. No Brasil esta participação da família tem oscilado entre $22 \%$ e $55 \%$ dos casos de LSV em crianças $^{1,2,13,14}$. Esses dados são parciais, pois como as pesquisas foram retrospectivas, em grande parte dos prontuários não havia descrição alguma de como a decisão de final de vida foi tomada. Num estudo argentino multicêntrico, publicado em 2003, foi observado que a participação da família ocorreu em $39 \%$ dos casos e em apenas $5 \%$ a LSV foi solicitada pela família ${ }^{15}$.

A pequena participação dos pais e, quando possível, dos próprios pacientes no processo decisório contribui para que o suporte de vida pleno seja mantido mesmo quando a situação é irreversível e a doença incurável ${ }^{16}$. Recentemente 0 Conselho Federal de Medicina emitiu a resolução $n^{\circ} 1.805 / 06$ que reforçou a responsabilidade do médico brasileiro em envolver as famílias além de dar definições do modelo de decisão compartilhada com respeito aos cuidados de final de vida ${ }^{17}$.

Uma dúvida freqüente em nosso meio refere-se ao "interesse" das famílias em participar efetivamente da decisão de limitar terapias ao seu filho com doença terminal, como ocorre no hemisfério norte ${ }^{18-21}$. Não existem razões para que se possa imaginar que os pais dos hemisférios norte e sul tenham comportamentos diferentes frente à morte eminente de seus filhos. A hi- 
pótese é que a diferença se encontra no preparo das equipes médicas para enfrentar este delicado problema. Assumir que as famílias brasileiras teriam "falta de interesse ou despreparo" para participar ativamente neste processo, além de ser discriminatório representa uma desculpa fácil para que médicos continuem a preservar uma posição paternalista.

Na América do Norte e no Reino Unido o desejo de participar é praticamente universal e o paternalismo é coisa de um passado remoto, o que foi expresso assim por um editorial do BMJ: "Paternalismo ou participação: os pacientes cresceram, e não tem mais volta!"22.

Nos vários estudos norte-americanos, $75 \%-85 \%$ das famílias entrevistadas desejavam ter a palavra final na decisão de LSV12,17,23. Muitas vezes isso chega ao extremo das famílias trazerem o assunto de cessar o tratamento mesmo antes de a equipe médica o fazer, como ficou demonstrado no estudo de Garros e col. ( $25 \%$ dos casos de LSV) ${ }^{6}$.

É evidente que a maioria das famílias prefere tomar sua decisão depois que a equipe assistencial expressa a sua recomendação. Meert e col. entrevistaram familiares de crianças depois da suas mortes na UTI e observaram que em $49 \%$ das ocasiões os pais tomaram a decisão de suspender o tratamento, em $29 \%$ os médicos, e em $15 \%$ a conduta foi compartilhada ${ }^{19,21}$.

$\mathrm{Na}$ Espanha estudo multicêntrico aponta que $28 \%$ das famílias entrevistadas relataram que não foram envolvidas na decisão, $41 \%$ afirmaram que a decisão foi compartilhada, $28 \%$ disseram que foi um acordo indireto e em $3 \%$ as famílias disseram que recusaram a aceitar a recomendação do médico, mas o tratamento foi assim mesmo retirado ou limitado. A discussão sobre o fim da vida foi iniciada pelo médico em $93 \%$ das vezes nesse estudo ${ }^{24}$.

Na França, $17 \%$ a $44 \%$ das famílias são envolvidas na decisão de final de vida. Os médicos franceses detêm papel preponderante na tomada de decisão. $\mathrm{O}$ argumento para esta prática se baseia no fato dos médicos considerarem que os pais de crianças internadas em UTIP têm sua capacidade de decisão prejudicada pelo estresse no qual se encontram. Neste caso, os médicos preferem proteger os pais da culpa de tomar uma decisão de final de vida irreversível para seus filhos. Esta atitude paternalista que desconsidera o princípio da autonomia é, evidentemente, contestada pelos intensivistas americanos e canadenses ${ }^{25-28}$.
A extrema ênfase do princípio da autonomia na América do Norte criou uma situação peculiar na Medicina, especialmente na área de intensivismo. A equipe médica tende a oferecer aos familiares dos pacientes pediátricos a oportunidade de escolher entre as opções de tratamentos existentes, quais poderiam ser retirados ou não oferecidos. O que se considera mais apropriado, ainda dentro do princípio ético da autonomia nos EUA, é o modelo de decisão compartilhada, onde o médico demonstra os prós e os contras de cada opção terapêutica e baseada no seu conhecimento e experiência profissional, recomenda a que seria mais adequada em cada caso ${ }^{29}$.

\section{O QUE AS FAMÍLIAS ESPERAM DA EQUIPE ASSIS- TENCIAL}

A principal exigência das famílias de crianças em final de vida é a oportunidade de participar ativamente na tomada de decisões pela equipe assistencial ${ }^{30}$.

Vários estudos feitos com familiares de pacientes que faleceram em UTIP demonstraram que apesar de todo sofrimento que representa a morte de um filho, o luto foi mais bem elaborado naqueles que acompanharam a criança durante todo o tratamento e participaram da discussão de planos de final de vida ${ }^{30}$.

Estudo envolvendo familiares de crianças que faleceram em uma UTIP norte-americana, as famílias deram grande valor aos seguintes aspectos: informação completa e honesta, pronto acesso ao médico responsável, ótima comunicação e coordenação no atendimento, apoio e empatia por parte da equipe de profissionais envolvidos no atendimento, preservação da integridade da relação entre pais e filho e respeito à religiosidade ${ }^{18}$.

Não são exigências de difícil execução, bastando apenas interesse do médico de priorizar o conforto do paciente e o respeito pela vontade da família. Em estudo realizado, entrevistando os pais, observou-se que as principais solicitações feitas foram ser úteis, estar informado de cada mudança clínica, ter certeza que o paciente não estava sofrendo e poder extravasar suas emoções e ansiedades ${ }^{30}$.

Ao avaliar os sentimentos dos pais que perderam seus filhos em UTIP observou-se que a maioria dos familiares gostaria de ter tido mais possibilidade de discutir sobre a doença de seu filho e as condutas que foram tomadas. Aqueles que conseguiram participar do processo de morte de seus filhos sentiram-se mais confortados ${ }^{30}$. 


\section{ROTINA DE FINAL DE VIDA EM UTIP}

Não existe uma fórmula única de como proceder nos últimos momentos de vida de um paciente pediátrico, porém é evidente que o passo inicial é o interesse do médico em oferecer uma morte mais digna e tranqüila para esta criança9.

No Brasil, como no restante da América Latina, não existem protocolos assistenciais para o atendimento de pacientes terminais, porém as UTIP de paises do hemisfério norte, desenvolvem treinamentos especiais para seus profissionais visando à melhoria na qualidade do atendimento do período que precede a morte. Na UTIP do Stollery Children's Hospital, por exemplo, 0 atendimento de pacientes terminais segue uma rotina. A equipe, composta pelo médico-assistente chefe, acompanhado de um residente da enfermeira responsável pelo paciente, da assistente social e do capelão (se a família assim desejar), se reúne com a família e em comum acordo decidem por limitar ou retirar o suporte de vida da criança. A decisão pode ser tomada em reunião formal, mas comumente exige duas a três reuniões. No caso de retirada de suporte, o médico oferece à família, a opção de permanecer o tempo que julgar necessário com seu filho fazendo as suas despedidas e rituais religiosos ${ }^{12,31}$.

Imediatamente após a retirada do suporte de vida ou a não oferta da terapia considerada fútil, o foco da equipe passa a ser o conforto do paciente e da família. A administração de analgésicos e sedativos é a intervenção terapêutica primordial. Se doses supraterapêuticas de morfina forem necessárias para garantir o conforto, isto será feito dentro do princípio ético do duplo efeito ${ }^{5,12,33}$. A família deve ser repetidamente orientada para que compreenda que o tratamento removido não mudará a evolução da doença e que a morte pode ocorrer imediatamente ou demorar minutos ou até dias, ficando garantidos os cuidados paliativos com a criança. $O$ objetivo maior do atendimento de pacientes terminais neste hospital canadense é que a família lembre-se dos últimos momentos com a sua criança como sendo cheios de paz, amor, carinho e dedicação.

\section{CONCLUSÃO}

A melhora na qualidade do atendimento de final de vida tornou-se uma prioridade ${ }^{5,8}$. No Brasil, tem-se conseguido aumentar consideravelmente a sobrevida de crianças internadas em UTIP; entretanto, pelos estudos realizados no nosso meio, pode-se afirmar que ainda há um longo caminho a percorrer para melhorar $o$ atendimento de crianças que vem a falecer de doenças irreversíveis.

Vários estudos têm demonstrado a importância da participação efetiva da família e da equipe assistencial no processo de LSV em crianças com doenças terminais ${ }^{18-21,31,34}$. Na América Latina ainda não foram realizados estudos avaliando a efetiva participação da família no processo de final de vida e o grau de satisfação dos pais. Somente após conhecer esta realidade é que se poderá definir a estratégia adequada para meIhorar a qualidade do atendimento a crianças internadas com doenças irreversíveis em UTI pediátricas do Brasil. Mas, sem dúvida alguma, ao desconsiderar a participação da família nesta decisão, os médicos podem estar optando por condutas que não atendem as expectativas, anseios e valores do paciente e de seus pais frente à morte eminente.

\section{REFERÊNCIAS}

01. Kipper DJ, Piva JP, Garcia PC et al - Evolution of the medical practices and modes of death on pediatric intensive care in southern Brazil. Pediatr Crit Care, 2005;6:258-263.

02. Lago PM, Piva J, Kipper D et al - Life support limitation at three pediatric intensive care units in southern Brazil. J Pediatr, 2005;81:111-117.

03. Feudtner C, Christakis DA, Zimmerman F et al - Characteristics of death occurring in children's hospitals: implications for supportive care services. Pediatrics, 2002;109:887-893.

04. Hinds PS, Schum L, Baker JN et al - Key factors affecting dying children and their families. J Palliat Med, 2005;8:(Suppl1):S70-S78.

05. Truog RD, Meyer EC, Burns JP - Toward interventions to improve end-oflife care in the pediatric intensive care unit. Crit Care Med, 2006;34:(Suppl11):S373-S379.

06. Garros D, Rosychuk RJ, Cox PN - Circumstances surrounding end of life in a pediatric intensive care unit. Pediatrics, 2003;112:e371-e379.

07. Carlet J, Thijs LG, Antonelli M et al - Challenges in end-of-life care in the ICU. Statement of the $5^{\text {th }}$ International Consensus Conference in Critical Care: Brussels, Belgium, April 2003. Intensive Care Med, 2004;30:770784.

08. Truog RD, Cist AF, Brackett SE et al - Recommendations for end-of-life care em the intensive care unit: The Ethics Committee of the Society of Critical Care Medicine. Crit Care Med, 2001;29:2332-2348.

09. Lago PM, Devictor D, Piva JP - End-of-life care in children: the Brazilian and the international perspectives. J Pediatr, 2007;83:(Suppl2):S109S116.

10. Mateos C, Lopez PR, Diaz JS et al - Cuidados al final de la vida en la unidad de cuidados intensivos pediátrica. Revisión de la bibliografía. Ann Pediatr, 2006;63:152-159.

11. Garros D - A "good" death in pediatric ICU: is it possible? J Pediatr, 2003;79:(Suppl2):S243-S254.

12. Provoost V, Cools F, Deconinck $\mathrm{P}$ et al - Consultation of parents in actual end-of-life decision-making in neonates and infants. Eur J Pediatr, 2006;165:859-866.

13. Carvalho PR, Rocha TS, Santo AE et al - Modes of death in the PICU of a tertiary care hospital. Rev Ass Med Brasil, 2001;47:325-331.

14. Tonelli HA, Mota JA, Oliveira JS - A profile of the medical conduct preceding child death at a tertiary hospital. J Pediatr, 2005;81:118-125.

15. Althabe M, Cardigni G, Vassallo JC et al - Dying in the intensive care unit: collaborative multicenter study about forgoing life-sustaining treatment in Argentine pediatric intensive care units. Pediatr Crit Care Med, 2003;4:164-169.

16. Lago PM, Piva J, Garcia PC et al - Brain death: medical management 
in seven Brazilian pediatric intensive care units. J Pediatr, 2007;83:133140.

17. Conselho Federal de Medicina. Resolução CFM 1.805/2006. Diário Oficial da União 2006;28:196.

18. Meyer EC, Ritholz MD, Burns JP et al - Improving the quality of end-oflife care in the pediatric intensive care unit: parents' priorities and recommendations. Pediatrics, 2006;117:649-657.

19. Coulter A, Entwistle V, Gilbert D - Sharing decisions with patients: is the information good enough? BMJ, 1999;318:318-322.

20. Sprung $\mathrm{CL}$, Cohen SL, Baras M et al - End-of-life practices in European intensive care units. The Ethicus Study. JAMA, 2003;290:790-797

21. Meert KL, Thurston CS, Sarnaik AP - End-of-life decision-making and satisfaction with care: parental perspectives. Pediatr Crit Care Med, 2000;1:179-185.

22. Coulter A - Paternalism or partnership? Patients have grown up-and there's no going back. BMJ, 1999;319:719-720.

23. Solomon MZ, Sellers DE, Heller KS et al - New and lingering controversies in pediatric end-of-life care. Pediatrics, 2005;116:872-883.

24. Devictor DJ, Nguyen DT - Forgoing life-sustaining treatments in children: a comparison between Northern and Southern European pediatric intensive care units. Pediatr Crit Care Med, 2004;5:211-215.

25. Esteban A, Gordo F, Solsona JF et al - Withdrawing and withholding life support in the intensive care unit: a Spanish prospective multi-centre observational study. Intensive Care Med. 2001:27:1744-1749.

26. Pochard F, Azoulay E, Chevret S et al - French Intensivists do not apply
American recommendations regarding decisions to forgo life-sustaining therapy. Crit Care Med, 2001;29:1887-1892.

27. Carnevale FA, Canoui P, Cremer $R$ et al - Parental involvement in treatment decision regarding their critically ill child: a comparative study of France and Quebec. Pediatr Crit Care Med, 2007;8:337-342.

28. Devictor DJ, Nguyen DT, Groupe Francophone de Reanimation et d'Urgences Pediatriques. Forgoing life-sustaining treatments: how the decision is made in French pediatric intensive care units. Crit Care Med, 2001;29:1356-1359.

29. Zawistowski CA, deVita MA - A descriptive study of children dying in the pediatric intensive care unit, after withdrawal of life sustaining treatment. Pediatr Crit Care Med, 2004;5:216-223.

30. Meert KL, Eggly S, Pollack M et al - Parents perspectives regarding a physician-parent conference after their child's death in the pediatric intensive care unit. J Pediatr, 2007;151:50-55.

31. Meert KL, Thurston CS, Briller SH - The spiritual needs of parents at the time of their child's death in the pediatric intensive care unit and during bereavement: a qualitative study. Pediatr Crit Care Med, 2005;6:420-427.

32. Devictor DJ - Are we ready to discuss spirituality with our patients and their families? Pediatr Crit Care Med, 2005;6:492-449.

33. Carter BS, Howenstein M, Gilmer MJ et al - Circumstances surrounding the deaths of hospitalized children: opportunities for pediatric palliative care. Pediatrics, 2004:114; e361-e366.

34. Solomon MZ, Sellers DE, Heller KS et al - New and lingering controversies in pediatric end-of-life care. Pediatrics, 2005;116:872-883. 